\title{
Drug use evaluation of opioid analgesics in pain management among patients with hematopoietic stem cell transplantation
}

\author{
Hyun Jin $\mathrm{Oh}^{1}$, So Yeon Hong ${ }^{1}$, Young Mi Jeong ${ }^{1}$, Kyung Suk Choi ${ }^{1}$, Eunsook Lee ${ }^{1}$, Euni Lee ${ }^{2}$, \\ Yu Jung Kim ${ }^{3}$, Soo-Mee Bang \\ ${ }^{1}$ Department of Pharmacy, Seoul National University Bundang Hospital, Seongnam, ${ }^{2}$ College of Pharmacy \& Research Institute of \\ Pharmaceutical Sciences, Seoul National University, Seoul, ${ }^{3}$ Division of Hematology and Medical Oncology, Department of Internal \\ Medicine, Seoul National University Bundang Hospital, Seongnam, Korea
}

p-ISSN 2287-979X / e-ISSN 2288-0011 https://doi.org/10.5045/br.2020.2020056 Blood Res 2020;55:151-158.

Received on March 26, 2020

Revised on July 15, 2020

Accepted on August 7, 2020

\section{Background}

Hematopoietic stem cell transplantation (HSCT) patients usually experience mucositis, musculoskeletal pain associated with high-dose chemotherapy, radiation, post-HSCT infection, or graft-versus-host disease. Pain management is important for the patients' quality of life. We evaluated appropriate opioid analgesic use in HSCT patients to propose effective pain management strategies.

\section{Methods}

A retrospective analysis was conducted using electronic medical records of adult patients with HSCT treated with opioids for moderate to severe pain at Seoul National University Bundang Hospital. The numeric rating scale (NRS) was used in pain management. NRS scores of 4-10 correspond to moderate to severe pain. Appropriate opioid analgesic use was evaluated following published cancer pain management guidelines.

\section{Results}

In total, 119 cases were evaluated, including 369 episodes of moderate to severe pain. Mucositis-related, musculoskeletal, and headache pain occurred in $62.6 \%, 25.8 \%$, and $6.0 \%$ of episodes, respectively. Frequently used opioids were intravenous tramadol (84.9\%), fentanyl patch (73.9\%), and intravenous morphine sulfate $(68.9 \%)$. Intravenous and topical administrations were used for mucosal pain. In total, $95.0 \%$ of patients received appropriate short-acting opioids for initial pain management, $80.5 \%$ received appropriate doses of short-acting opioids, appropriate opioids dose adjustment was done after first assessment in $95.5 \%$ of patients, and $85.6 \%$ were converted to appropriate long-acting opioids.

\section{Conclusion}

Short-acting opioid analgesic use for initial pain management and dose adjustment after assessment were appropriate. However, initial and conversion dosages recommended by guidelines may be difficult to implement considering the severity of HSCT patients. Pain management guidelines specific for HSCT patients should be developed in the future.

Key Words Hematopoietic stem cell transplantation, Opioid, Analgesics, Pain management

\section{INTRODUCTION}

Hematological malignancies are characterized by the abnormal differentiation and proliferation of bone marrow or blood cells of the immune system, and major diseases include leukemia, lymphoma, multiple myeloma, and myelodys- plastic syndrome (MDS) [1]. In Korea, hematological malignancies are a relatively rare form of cancer that accounts for less than $6 \%$ of all cancers in the Korean population [2]. Nonetheless, with the aging population, increased westernized dietary habits, and the effects of environmental factors, the number of patients with hematological malignancies is steadily increasing [3]. 
Chemotherapy is the main treatment option for hematological malignancies, and hematopoietic stem cell transplantation (HSCT) is often used as the ultimate treatment for cure of the disease. HSCT is divided into allogeneic and autologous transplants. In allogeneic transplants, healthy hematopoietic stem cells from a donor are injected to replace the defective hematopoietic system. Conversely, autologous transplant involves aggressive chemotherapy to eradicate residual malignant cells, followed by transplantation of the patient's own hematopoietic stem cells. This approach requires conditioning regimens including high-dose chemotherapy and radiation $[4,5]$.

Throughout this process, the majority of patients undergoing HSCT experience extreme levels of pain associated with the various systemic side-effects of the treatment [6-8]. Because of the aggressive and intense nature of the therapeutic regimen, the severity and pattern of symptoms are much more severe and complex than those in patients with other cancers $[9,10]$. More specifically, the digestive mucosal membrane is first to be affected by cytotoxic treatment. Inflammation and hemorrhage in these digestive organs result in the frequent onset of stomatitis and sore throat [11]. In addition, pain in the skeletal system and muscles caused by granulocyte colony-stimulating factor (G-CSF) may occur as well [12]. The painful symptoms associated with these therapeutic regimens result in a consequential loss of appetite and sleep disturbance in patients, leading to negative outcomes such as post-transplantation weight loss and delayed recovery $[13,14]$. Nevertheless, $80-90 \%$ of these painful symptoms can be adequately managed through medication according to the principles of pain management, and appropriate evaluation of pain and drug treatment are therefore crucial factors in improving patients' clinical outcomes and quality of life [15, 16].

According to the previous study by Wardley et al. [17], approximately $67 \%$ of patients with HSCT develop severe mucositis accompanied by intraoral pain, and these patients may have great difficulties consuming food because of ulcers. Therefore, it is important to manage and provide appropriate interventions for this condition, and evaluation of the adequacy of the opioid treatment used should precede appropriate drug-based interventions.

The Division of Hematology-Oncology at Seoul National University Bundang hospital (SNUBH) provides medical services that include the management of conditioning regimens and interventions for patients with HSCT and educates patients about drug use. In this study, we aimed to evaluate the adequacy of opioid analgesic used for pain management and to suggest new clinical roles of pharmacists for more effective pain management.

\section{MATERIALS AND METHODS}

\section{Study population}

The subjects included were adult patients ( $\geq 19$ years old) undergoing HSCT and receiving opioid analgesics for moder- ate to severe pain that developed after HSCT at SNUBH between June 2015 and May 2018. Patients who were prescribed opioid analgesics for other reasons than pain (i.e., additional procedures) and those who received opioid analgesics through infusion pumps were excluded.

\section{Data collection}

Using Electronic Medical Records (EMR), we retrospectively collected subjects' data, including general information (gender, age, diagnosis, type of transplantation, and conditioning regimen before HSCT), information on initial pain of moderate or severe degree, and information regarding the type and dose of opioid analgesics used. In addition, pain severity was re-evaluated, and changes in pain severity after the use of opioid analgesics were also recorded. The level of pain was quantified using a numerical rating scale (NRS) to quantify pain severity based on the patient's subjective response $[18,19]$. Initial pain assessments were limited to $\leq 5$ assessments per patient. Pain was re-evaluated after injection of short-acting opioid analgesics to assess if appropriate dosage adjustments were made based on the changes in pain severity. Repeated injection of the same dosage was considered appropriate in cases of reduced pain and a $50-100 \%$ increase of the original dosage was considered appropriate in cases of persistent or increased pain. After repeated injection of short-acting opioid analgesics according to the outcomes of pain re-evaluation, we assessed whether conversion to sustained-release opioid analgesics based on a $24 \mathrm{hr}$ dosage was appropriately conducted by referring to the equianalgesic and fentanyl patch conversion charts (Supplementary Table 1). In this study, opioid analgesics were defined as any drug that pharmacologically acts on opioid receptors and exerts an analgesic effect.

\section{Evaluation categories}

To evaluate the adequacy of drug usage, we assessed complaints of pain symptoms and use of opioid analgesics in

Table 1. Drug use evaluation items and criteria for appropriate use of opioid analgesics.

\begin{tabular}{|c|c|}
\hline Evaluation items & Criteria \\
\hline Initial drug selection & $\begin{array}{l}\text { Using SA opioid }{ }^{\text {a) }} \text { for initial pain that is } \\
\text { moderate to severe (NRS } 4-10 \text { ) }\end{array}$ \\
\hline Initial dose selection & $\begin{array}{l}\text { 5-15 mg oral SA morphine sulfate or } \\
\text { equivalent }\end{array}$ \\
\hline Dosage adjustment & $\begin{array}{l}\text { Decreased pain: repeat the same dose } \\
\text { Unchanged or increased pain: increase } \\
\text { dose by } 50-100 \%\end{array}$ \\
\hline Dosage conversion & $\begin{array}{l}\text { LA opioid }{ }^{\text {b) }} \text { corresponds to } 50-100 \% \text { total } \\
\text { daily requirements of SA opioid dosage }\end{array}$ \\
\hline \multicolumn{2}{|c|}{$\begin{array}{l}\text { a) SA opioid: fentanyl buccal, oral tramadol, oral oxycodone, } \\
\text { intravenous tramadol, intravenous morphine sulfate. } \\
\text { b) LA opioid analgesics: topical fentanyl patch, oral oxycodone/ } \\
\text { naloxone. } \\
\text { Abbreviations: LA, long-acting; NRS, numerical rating scale; SA, } \\
\text { short-acting. }\end{array}$} \\
\hline
\end{tabular}


the cohort of study subjects. In addition, we analyzed the correlation between the site of pain and administration route of opioid analgesics. Initial pain management using short-acting opioid analgesics, dosage of short-acting opioid analgesics, adjustments in the dosage after re-evaluation, and conversion ratio to sustained-release opioid analgesics were evaluated. For all evaluations, we referred to the 2015 guidelines on cancer-related pain management by the Ministry of Health and Welfare in Korea and the 2018 adult cancer pain guidelines by the National Comprehensive Cancer Network (NCCN; Table 1) [18, 19].

\section{Analysis methods}

Data analysis was performed using Microsoft Office Excel 2016 (Microsoft, Redmond, WA, USA). The patients' characteristics are reported as means \pm standard deviation, frequency, and percentage (\%) values. The adequacy of each evaluation category was analyzed after converting the number of patients to percentage (\%) values.

\section{Ethics statement}

This study was approved by the Institutional Review Board (IRB) of SNUBH and the requirement to obtain informed consent was waived because of the retrospective nature of this study (IRB no. B-1809-492-111; date of waiver: September 3, 2018).

\section{RESULTS}

\section{Patient characteristics}

Between June 2015 and May 2018, 120 adult patients ( $\geq 19$ years old) underwent HSCT at SNUBH and were treated with opioid analgesics for moderate to severe post-transplantation pain. Among these, eight patients who received opioid analgesics via infusion pumps were excluded. Seven of the remaining 112 patients underwent HSCT twice during the study period. Each of these two HSCT cases was included as a separate case in this study, and ultimately there were 119 cases included in this study. There were 67 (56.3\%) male patients and 52 (43.7\%) female patients, and the mean age of patients was $47.5 \pm 12.4$ years. The most frequent tumor type was acute myeloid leukemia (AML) with 33 cases (27.7\%), followed by non-Hodgkin lymphoma (23.5\%), acute lymphocytic leukemia (ALL, 12.6\%), and multiple myeloma (11.8\%). There were 80 cases of allogeneic HSCT (67.2\%) and 39 cases of autologous HSCT (32.8\%; Table 2).

\section{Occurrence of pain and use of opioid analgesics}

Analysis of pain occurrence in HSCT patients revealed that there were 369 episodes in which the patient experienced moderate to severe pain. The most common type of pain was mucositis-related pain from the oral cavity to the anus (231 episodes, 62.6\%), followed by musculoskeletal system pain (95 episodes, 25.8\%), and headaches (22 episodes, $6.0 \%$ ). The opioid analgesics most commonly used to alleviate pain were tramadol injection (99 episodes, $28.0 \%$ ), followed by fentanyl patch (86 episodes, $24.3 \%$ ), and morphine sulfate injection (80 episodes, $22.6 \%$ ).

\section{Outcome of adequacy evaluation}

Initial pain management using short-acting opioid analgesics: The adequacy of initial pain management using short-acting opioid analgesics was assessed according to whether or not the patient had used short-acting opioid analgesics for pain management (NRS score $\geq 4$ ) during the hospitalization period. The types of opioid analgesics used for initial pain management included buccal fentanyl, oral tramadol, oral oxycodone, intravenous tramadol, and intravenous morphine sulfate. The distribution of short-acting analgesics used was different according to type of pain. For patients with mucositis-related pain, intravenous morphine sulfate $(58.1 \%)$, intravenous tramadol $(32.4 \%)$, oral tramadol $(5.4 \%)$, and oral oxycodone $(4.1 \%)$ were used. The route of administration was intravenous $(90.5 \%)$ and oral $(9.5 \%)$. For patients with musculoskeletal pain, intravenous morphine sulfate $(40.6 \%)$, oral oxycodone $(37.5 \%)$, intravenous tramadol $(9.4 \%)$, oral tramadol (6.3\%), and buccal fentanyl (6.2\%) were used. The route of administration was intravenous (50.0\%), oral (43.8\%), and buccal (6.2\%). These short-acting opioid analgesics were used in 113 of 119 cases, and thus adequate pain management was observed in $95 \%$ of all cases (Table $3)$. The other 6 cases used long-acting formulations as the first opioid analgesic for initial pain management during hospitalization. All 6 cases had autologous hematopoietic stem cell transplantation, and 2 of these cases had used fentanyl patches due to side effects of morphine and tramadol.

Dosage of short-acting opioid analgesics: The dosage of short-acting opioid analgesics used was evaluated by comparison of the actual dosage of short-acting opioid analgesics used for initial pain management and the dosage suggested by guidelines or the equianalgesic chart (Supplementary

Table 2. Clinical characteristics of the study subjects $(N=119)$.

\begin{tabular}{llc}
\hline \multicolumn{1}{c}{ Characteristics } & N of cases (\%) \\
\hline \multirow{2}{*}{ Age $(y r$, mean \pm SD) } & $47.5 \pm 2.4$ \\
Sex & Male & $67(56.3)$ \\
\multirow{4}{*}{ Diagnosis } & Female & $52(43.7)$ \\
& Acute myeloid leukemia & $33(27.7)$ \\
& Non-Hodgkin lymphoma & $28(23.5)$ \\
& Acute lymphoblastic leukemia & $15(12.6)$ \\
& Multiple myeloma & $14(11.8)$ \\
& Myelodysplastic syndrome & $11(9.2)$ \\
& Aplastic anemia & $7(5.9)$ \\
& Hodgkin lymphoma & $4(3.4)$ \\
HSCT type & Other $\left.{ }^{a}\right)$ & $7(5.9)$ \\
& Allogenic & $80(67.2)$ \\
& Autologous & $39(32.8)$ \\
\hline
\end{tabular}

${ }^{\text {a) }}$ Other diagnoses included amyloidosis, acute biphenotypic leukemia, two cases of chronic myelomonocytic leukemia, germ cell tumor, and two cases of hemophagocytic lymphohistiocytosis. Abbreviation: HSCT, hematopoietic stem cell transplantation. 
Table 3. Drug use evaluation of opioid analgesics.

\begin{tabular}{|c|c|c|c|c|}
\hline \multirow{2}{*}{ Evaluation items } & \multicolumn{2}{|l|}{ Appropriate } & \multicolumn{2}{|l|}{ Inappropriate } \\
\hline & Criteria & $\mathrm{N}(\%)$ & Criteria & $\mathrm{N}(\%)$ \\
\hline Initial drug selection & SA opioid analgesics & $113(95.0)$ & LA opioid analgesics & $6(5.0)^{a)}$ \\
\hline Initial dose selection & Recommended dosage $^{\text {b) }}$ & $91(80.5)$ & Higher dosage $^{c)}$ & $22(19.5)$ \\
\hline \multirow[t]{2}{*}{ Dosage adjustment } & Same dose with reduced pain & $97(86.6)$ & Suboptimal dosage increase ${ }^{\mathrm{e}}$ & $5(4.5)$ \\
\hline & Sufficient dosage increase $^{\text {d) }}$ & $10(8.9)$ & & \\
\hline Dosage conversion & $50-100 \%$ of TDD & $83(85.6)$ & $>100 \%$ of TDD & $14(14.4)$ \\
\hline
\end{tabular}

Table 1). Of the 113 cases in which short-acting opioid analgesics were used, analysis outcomes showed that there were 91 cases $(80.5 \%)$ of appropriate dosage and 22 cases (19.5\%) of inappropriate dosage (Table 3). In the 22 cases of inappropriate dosage, the patients were administered higher doses than those recommended in the guidelines. More specifically, $100 \mathrm{mg}$ tramadol injection was used in 21 cases and $10 \mathrm{mg}$ morphine injection was used in one case of initial pain management. According to the guidelines, 5-15 mg oral short-acting morphine sulfate or an equivalent dose of opioid analgesics are appropriate for initial pain management, but $100 \mathrm{mg}$ intravenous tramadol and $10 \mathrm{mg}$ intravenous morphine sulfate exceed the $15 \mathrm{mg}$ oral short-acting morphine sulfate dose or an equivalent dose.

Dose adjustment after re-evaluation: One case in which the patient refused the continuous use of short-acting opioid analgesics was excluded from evaluation. Evaluation outcomes demonstrated that in 107 of 112 cases (95.5\%), analgesic dosage adjustment after re-evaluation complied with the guidelines. In the remaining 5 cases (4.5\%), the patients exhibited persistent or worsened pain after re-evaluation, yet the increase in dosage was $<50 \%$, which was below recommendations (Table 3 ).

Conversion to sustained-release opioid analgesics: The converted dosage was appropriate in $83(85.6 \%)$ of the 97 cases in which patients received sustained-release medication. There were 14 cases (14.4\%) in which patients received sustained-release medication with converted dosage exceeding $100 \%$ of the recommended $24 \mathrm{hr}$ short-acting medication dose. In seven of these 14 cases, the $24 \mathrm{hr}$ short-acting medication dose should be $5 \mathrm{mg}$ morphine injection, but patients received $12 \mu \mathrm{g} / \mathrm{h}$ fentanyl patches (equivalent to $10 \mathrm{mg}$ morphine injection) because of severe mucositis-related difficulties in consuming oral sustained-release tablets. In addition, there were 2 cases of accompanying side-effects from morphine or tramadol injections, and 5 cases of overdose ( $>100 \%$ of the $24 \mathrm{hr}$ short-acting medication dose) without severe mucositis or side effects from short-acting medication (Table 3).

\section{DISCUSSION}

This study aimed to analyze treatment-related pain in patients with HSCT and consequent use of opioid analgesics in an attempt to recommend more appropriate medication-based strategies.

The most common forms of pain experienced by patients with HSCT were pain in the oral cavity, throat, and abdomen, which is caused by mucositis of the digestive system. These symptoms hinder food intake after treatment, resulting in weight loss and delayed recovery. Thus, active pain management is closely related to successful treatment outcome [20]. However, the use of oral analgesics for pain management may be hampered in patients who have difficulties with oral intake of medication associated with severe pain and vomiting. Consequently, in this study, we observed that the frequency of non-oral opioid analgesic use (i.e., injections and patches) for pain management was higher than that of oral analgesics.

Appropriate use of opioid analgesics for cancer-related pain management is a crucial factor for the patient's quality of life. The Korean guidelines on cancer-related pain management and the adult cancer pain guidelines by the NCCN both suggest the use of opioid analgesics for effective moderate to severe pain management. Moreover, these guidelines recommend that short-acting oral medication or injections should be used in initial pain management and to repeatedly evaluate the appropriate dosage based on the patient's symptoms. In addition, the guidelines recommend to switch to sustained-release medication once the $24 \mathrm{hr}$ (daily) dosage is determined [18, 19].

According to a previous study, the mean NRS score was reduced from 6.5 to 2.6 after analgesic use for cancer-related pain that was well managed using the appropriate dosage of opioid analgesics [21]. Furthermore, greater adherence to guidelines resulted in a greater number of patients receiving appropriate pain management within a shorter time period [22]. In this study, we evaluated the adequacy of opioid analgesic use according to guidelines, and demonstrated the following: initial pain management using short-acting opioid analgesics was adequate in $95.0 \%$ of cases and dosage was 
adequate in $80.5 \%$ of cases, dosage adjustment was adequate after re-evaluation in $95.5 \%$ of cases, and conversion to sustained-release opioid analgesics was adequate in $85.6 \%$ of cases. Although the adequacy of initial pain management using short-acting opioid analgesics and dosage adjustments based on re-evaluation was high ( $\geq 95 \%)$, the adequacy of the initial dosage of short-acting medication and conversion to sustained-release medication was relatively lower.

All cases using long-acting opioid analgesics for initial pain management were autologous hematopoietic stem cell transplantation. This is thought to be due to the fact that most patients who have been using long-acting formulations for underlying pain disease prior to hospitalization have not been treated with additional short-acting formulations for sudden pain during hospitalization.

We also evaluated the adequacy of the initial dosage of short-acting opioid analgesics and observed that in 21 of 22 cases in which an inappropriate dosage was used, 100 mg tramadol injection was administered. When converted using the equianalgesic chart in the guidelines, $100 \mathrm{mg}$ tramadol injection corresponds to $30 \mathrm{mg}$ oral morphine or 10 $\mathrm{mg}$ morphine injection. As the guidelines suggest that the initial dosage of short-acting morphine should be 5-15 mg (oral administration), $100 \mathrm{mg}$ tramadol injection was considered an inappropriate initial dose. Nevertheless, according to the permitted use of tramadol in Korea, 50-100 mg tramadol may be injected intravenously once per day, and repeated injections may be carried out every $4-5 \mathrm{~h}$, up to $400 \mathrm{mg}$ per day [23]. This suggests that $100 \mathrm{mg}$ tramadol injection is an appropriate clinical dose. In addition, tramadol is a synthetic opioid analog whose affinity for the $\mu$ receptor is 6,000 times weaker than that of morphine and whose potency is 6- to 20 -fold lower than that of morphine [24, 25].

Of 97 cases in which sustained-release medication was used, analysis of the conversion to sustained-release opioid analgesics demonstrated that $83(85.6 \%)$ involved $24 \mathrm{hr}$ doses equivalent to $50-100 \%$ of the $24 \mathrm{hr}$ short-acting analgesic dose. However, there were 14 cases $(14.4 \%)$ in which the dose exceeded $100 \%$ of the $24 \mathrm{hr}$ dose of short-acting analgesics, and 13 of these 14 cases involved the patients using fentanyl patches. Of the 13 cases using fentanyl patches, 9 were cases that used fentanyl patches due to difficulties in taking oral medication because of mucositis-related pain. The other 4 were cases of musculoskeletal pain and headache. The distribution of HSCT types for the 13 cases was 9 allogenic HSCT and 5 autologous HSCT.

In patients with severe mucositis caused by aggressive cytotoxic anticancer agents administered during the conditioning regimen, fentanyl patch is a more convenient method of pain management than morphine injection and is a better option than sustained-release oral medication because of the severe oral pain experienced by these patients. Recent studies have indicated that fentanyl patch is an effective tool for the management of pain associated with oral mucositis caused by chemotherapy and is also a noninvasive application compared to injections, especially in immunosuppressed patients [26, 27]. However, the lowest-dose fentanyl patch that can be used is $12 \mu \mathrm{g} / \mathrm{h}$, which is equivalent to $10 \mathrm{mg}$ morphine injection. Therefore, if the $24 \mathrm{hr}$ dosage of short-acting medication is $<10 \mathrm{mg}$ morphine injection, the use of a fentanyl patch will be considered an inappropriate converted dosage in all cases. For appropriate pain management in patients who cannot swallow tablets and require $<10$ mg morphine, a non-oral sustained-release medication with dosage lower than fentanyl $12 \mu \mathrm{g} / \mathrm{h}$ is required. Among opioid analgesics commercially available in Korea, the buprenorphine patch is a partial agonist of the $\mu$ opioid receptor, and its lowest available dosage is $5 \mu \mathrm{g} / \mathrm{h}$, which corresponds to $5 \mathrm{mg}$ morphine injection. Thus, this patch is an effective and safe method for pain management in patients who require relatively low-dose analgesics but have difficulties in swallowing tablets [28, 29]. However, in Korea, the use of the buprenorphine patch was approved only for relief from moderate to severe chronic pain that does not respond to non-opioid analgesics, and thus its use in HSCT patients will likely be limited [30]. Therefore, the guidelines should be changed to recommend IV injection of an appropriate dose of morphine over a $24 \mathrm{hr}$ period in patients receiving sustained-release medication that exceeds the recommended short-acting medication dose. Alternatively, if injection is not an option and fentanyl patches must be used, active monitoring against possible side effects (i.e., sedation/drowsiness, delirium from sudden increase, diarrhea, nausea/vomiting, and other neurological side effects from high dose administration) is required [18]. Although we attempted to compare our study outcomes with the results of other similar studies based on the Korean guidelines for cancer-related pain management, there were no studies that evaluated the adequacy of doses used when converting from short-acting to sustained-release medication.

Because of the retrospective design of this study, persistent evaluation and monitoring of pain were difficult to perform. Thus, only a few procedures suggested in the guidelines were selected for evaluation. Furthermore, we could not perform additional analyses of potential side effects in relation to the results that were considered inappropriate in the study and did not evaluate the analgesic requirements according to conditioning, age, weight, and neutropenia prior to conditioning. Despite these limitations, we successfully identified the cause of moderate to severe pain in adult patients with HSCT, investigated the use of opioid analgesics for pain management, and assessed the adequacy of opioid analgesic use. Based on these findings, cases in which adequacy was relatively low may be improved in the future and guidelines on the management of opioid analgesics and pain in patients with HSCT may be amended.

This study evaluated the adequacy of opioid analgesic use for pain management in patients with HSCT. The study outcomes indicated that initial pain management using short-acting opioid analgesics and dosage adjustment after re-evaluation of pain were appropriate, although the adequacy of the initial dose of short-acting medication and conversion to sustained-release medication was relatively 
low. Management of pain that occurs after a conditioning regimen for HSCT is crucial to improving the patient's quality of life. However, as the recommended use of opioid analgesics outlined in the guidelines may not be applicable to every different clinical situation, the establishment of pain management guidelines for patients with HSCT and continuous education regarding these guidelines are needed. The pharmacist should actively monitor the patient's pain and history of drug use in order to determine the appropriate use of opioid analgesics in the process of pain control in HSCT patients.

\section{Authors' Disclosures of Potential Conflicts of Interest}

No potential conflicts of interest relevant to this article were reported.

\section{REFERENCES}

1. National Cancer Institute. NCI dictionary cancer terms: hematologic cancer. Bethesda, MD: National Cancer Institute, 2018. (Accessed June 10, 2018, at https://www.cancer.gov/ publications/dictionaries/cancer-terms/def/hematologic-cancer).

2. Korean Statistical Information Service. 5 years prevalence number and relative frequency of 61 cancer types (2017). Daejeon, Korea: Statistics Korea, 2019. (Accessed May 30, 2019, at http:// kosis.kr/statHtml/statHtml.do?orgId=117\&tblId=DT_117N_A0 0121\&conn_path=I2).

3. Lee JJ, Ahn JS. Recently increasing hematologic diseases in Korea. Korean J Med 2010;78:557-63.

4. Hatzimichael E, Tuthill M. Hematopoietic stem cell transplantation. Stem Cells Cloning 2010;3:105-17.

5. Gyurkocza B, Sandmaier BM. Conditioning regimens for hematopoietic cell transplantation: one size does not fit all. Blood 2014;124:344-53.

6. Larsen J, Nordström G, Ljungman P, Gardulf A. Symptom occurrence, symptom intensity, and symptom distress in patients undergoing high-dose chemotherapy with stem-cell transplantation. Cancer Nurs 2004;27:55-64.

7. Anderson KO, Giralt SA, Mendoza TR, et al. Symptom burden in patients undergoing autologous stem-cell transplantation. Bone Marrow Transplant 2007;39:759-66.

8. Niscola P, Arcuri E, Giovannini M, et al. Pain syndromes in haematological malignancies: an overview. Hematol J 2004;5: 293-303.

9. Martino M, Morabito F, Console G, et al. Differences in transplant-related complications between hematologic malignancies and solid tumors receiving high-dose chemotherapy and autologous peripheral blood stem cell transplantation. Tumori 2003;89:385-90.

10. Chapko MK, Syrjala KL, Schilter L, Cummings C, Sullivan KM. Chemoradiotherapy toxicity during bone marrow transplantation: time course and variation in pain and nausea. Bone Marrow Transplant 1989;4:181-6.

11. Niscola P, Romani C, Cupelli L, et al. Mucositis in patients with hematologic malignancies: an overview. Haematologica 2007;92:
222-31.

12. Bassi S, Stroppa EM, Moroni CF, et al. Safety and efficacy of granulocyte colony-stimulating factor biosimilars in engraftment after autologous stem cell transplantation for haematological malignancies: a 4-year, single institute experience with different conditioning regimens. Blood Transfus 2015;13:478-83.

13. Cohen MZ, Rozmus CL, Mendoza TR, et al. Symptoms and quality of life in diverse patients undergoing hematopoietic stem cell transplantation. J Pain Symptom Manage 2012;44:168-80.

14. Haverman TM, Raber-Durlacher JE, Rademacher WMH, et al. Oral complications in hematopoietic stem cell recipients: the role of inflammation. Mediators Inflamm 2014;2014:378281.

15. Park JM. Cancer pain. In: Korean Society of Anesthesiologists. Anesthesiology and pain medicine. Seoul, Korea: Ryo Moon Gak, 2010:379-98.

16. Paice JA, Ferrell B. The management of cancer pain. CA Cancer J Clin 2011;61:157-82.

17. Wardley AM, Jayson GC, Swindell R, et al. Prospective evaluation of oral mucositis in patients receiving myeloablative conditioning regimens and haemopoietic progenitor rescue. Br J Haematol 2000;110:292-9.

18. National Cancer Center. Cancer pain management guideline. 6th ed. Goyang, Korea: National Cancer Center, 2018. (Accessed June 1, 2018, at https://www.cancer.go.kr/docview/preview.do?uuid= 718e6e53-b442-471e-b884-b49724a4a011.pdf).

19. National Comprehensive Cancer Network. Clinical Practice Guidelines in Oncology: adult cancer pain. Version 1 (2018). Plymouth Meeting, PA: National Comprehensive Cancer Network, 2018. (Accessed June 1, 2018, at https://www.nccn. org/professionals/physician_gls/default.aspx\#pain).

20. Gabriel DA, Shea T, Olajida O, Serody JS, Comeau T. The effect of oral mucositis on morbidity and mortality in bone marrow transplant. Semin Oncol 2003;30(6 Supp1 18):76-83.

21. Park SM, Han SH, Yoo SH, Lee YH, Lee HS, Oh JM. Evaluation of the adequacy of pain control using opioid analgesics in palliative ward patients using Cancer Pain Management Guidelines. J Kor Soc Health-Syst Pharm 2012;29:281-7.

22. Kim HT, Koh KW, Kim YM, et al. Pain management based on NCCN Guideline in patients with lung cancer. Tuberc Respir Dis 2009;67:221-5.

23. Ministry of Food and Drug Safety. Medicines patent information: tramadol hydrochloride. Cheongju, Korea: Ministry of Food and Drug Safety, 2018. (Accessed January 28, 2019, at https://nedrug. mfds.go.kr/pbp/CCBBB01/getItemDetail?itemSeq=198500825).

24. Park SS. Cancer pain management - opioids. J Korean Med Assoc 2010;53:250-7.

25. Lehmann KA. Tramadol for the management of acute pain. Drugs 1994;47(Suppl 1):19-32.

26. Xing SZ, Zhang Y. Efficacy and safety of transdermal fentanyl for the treatment of oral mucositis pain caused by chemoradiotherapy in patients with esophageal squamous cell carcinoma. Support Care Cancer 2015;23:753-9.

27. Guo SP, Wu SG, Zhou J, et al. Transdermal fentanyl for pain due to chemoradiotherapy-induced oral mucositis in nasopharyngeal cancer patients: evaluating efficacy, safety, and improvement in quality of life. Drug Des Devel Ther 2014;8:497-503.

28. Kress HG. Clinical update on the pharmacology, efficacy and 
safety of transdermal buprenorphine. Eur J Pain 2009;13:219-30.

29. Electronic Medicines Compendium. Summary of product characteristics: buprenorphine. Leatherhead, UK: Datapharm Communications Limited, 2018. (Accessed January 28, 2019, at https://www.medicines.org.uk/emc/product/9797/smpc).
30. Ministry of Food and Drug Safety. Medicines patent information: buprenorphine. Cheongju, Korea: Ministry of Food and Drug Safety, 2018. (Accessed January 28, 2019, at https://nedrug.mfds. go.kr/pbp/CCBBB01/getItemDetail?itemSeq=200410877). 
Supplementary Table 1. Relative potencies of opioid agents.

Oral and parenteral opioid equivalence

\begin{tabular}{cccc}
\hline Opioid agonist & Parenteral dose & Oral dose & Factor (IV to PO) \\
\hline Morphine & $10 \mathrm{mg}$ & $30 \mathrm{mg}$ & 3 \\
Oxycodone & - & $15-20 \mathrm{mg}$ & - \\
Tramadol & $100 \mathrm{mg}$ & $300 \mathrm{mg}$ & 3 \\
\hline
\end{tabular}

Conversion from oral and parenteral morphine to transdermal fentanyl

\begin{tabular}{ccc}
\hline Fentanyl patch & Intravenous morphine/24 h & Oral morphine/24 h \\
$12 \mu \mathrm{g} / \mathrm{h}$ & $10 \mathrm{mg}$ & $30 \mathrm{mg}$ \\
$25 \mu \mathrm{g} / \mathrm{h}$ & $20 \mathrm{mg}$ & $60 \mathrm{mg}$ \\
\hline
\end{tabular}

Abbreviations: IV, intravenous; PO, per oral. 